\title{
Double-Pomeron and Two-Photon Processes at RHIC
}

\author{
S. U. Chung, D. P. Weygand and H. J. Willutzki
}

Physics Department, Brookhaven National Laboratory, Upton, NY 11973, U.S.A.

\begin{abstract}
Double-Pomeron processes have been shown to be an important and novel source of hadron production at moderate energies at the ISR. ${ }^{1-6}$ These processes are expected to provide glue-rich hadrons from $1 \mathrm{GeV}$ to $10 \mathrm{GeV}$ or more, encompassing the states consisting of $u, d, s$ and $b$ quarks. The double-pomeron cross sections ${ }^{7-8}$ for central hadroproduction are calculated for $p \times p$ and $A u \times A u$ at RHIC.

Two-photon production of hadrons in the central region begins to dominate or at least become comparable to the double-Pomeron processes as the $Z$ of the beams increases from $p$ to $A u$. Since photons couple to charge, these hadroproductions involve mainly quarkonia and multiquark states. Therefore, a comparative study of these processes is expected to provide new insights into the constituents of hadronic matter. The two-photon processes are calculated following the recipe given by Cahn and Jackson ${ }^{9}$.

The paper starts out out with a thorough discission of the relevant kinematics, phase space and Regge amplitudes.
\end{abstract}

This manuscript has been authored under contract number DE-AC02-76CH00016 with the II.S. Department of Energy. Accordingly, the U.S. Goverument retains a non-exclusive, rovaltr-free license to publish in reproduce the published form of this contribution, or allow others to do so, for U.S. Cioverninent purpuses.

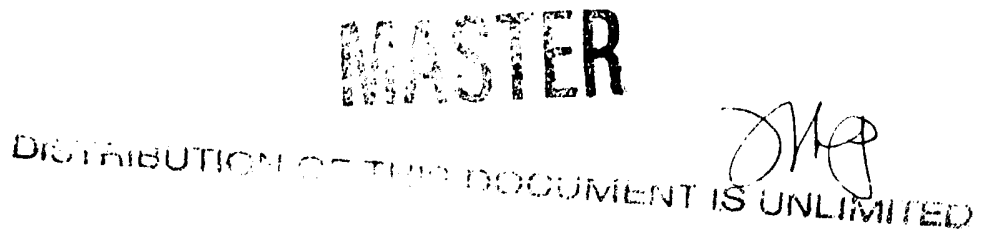




\section{Kinematics}

In this section are collected relevant kinematical variables for double-Pomeron exchange (DPE) reactions. This represents a concise update of an Internal Report by S. U. Chung ${ }^{10}$. Many of the approximations introduced in this note are checked against a mode of run at RHIC of $p \times p$ at $100 \mathrm{GeV} / c$, as an example. It can be shown that all the approximate formulas hold also for heavy-ion collisions, e.g. $A u \times A u$ at $100 \mathrm{GeV} / \mathrm{u}$ (per nucleon).

Consider a reaction

$$
a+b \rightarrow 1+3+2
$$

where a, b, 1 and 2 are initial and final protons and 3 represents the central hadronic system (see Fig. 1). The four-momenta may be expressed as

$$
\begin{aligned}
& p_{a}=(E, \overrightarrow{0}, k) \\
& p_{b}=(E, \overrightarrow{0},-k) \\
& p_{i}=\left(E_{i}, \vec{q}_{i}, k_{i}\right), \quad i=1,2,3
\end{aligned}
$$

where the second and the third elements are 2-dimensional transverse and 1-diniensional longitudinal components, respectively. Whenever possible, subscripts a and $b$ will be dropped from the initial protons. Thus, the magnitude of the initial proton momenta will be written $p_{a}=p_{b}=p$ and $E_{a}=E_{b}=E$.

These variables are given simple expressions in terms of the rapidity $y$ and the transverse mass $\tilde{n}$,

$$
\begin{aligned}
E & =m \cosh y \\
k & =m \sinh y, \quad y>0 \\
E_{i} & =\tilde{m}_{i} \cosh y_{i}, \quad i=1,2,3 \\
k_{i} & =\tilde{m}_{i} \sinh y_{i}, \quad y_{1}>0, \quad y_{2}<0 \\
\tilde{m}_{i} & =\sqrt{q_{i}^{2}+m^{2}}, \quad i=1,2 \\
\bar{m}_{3} & =\sqrt{q_{3}^{2}+m_{3}^{2}}
\end{aligned}
$$


where $m$ is the mass of the beam particles and $m_{3}$ is the effective mass of the central system. The rapidity of the central system can be expressed as

$$
\begin{aligned}
y_{3} & =\frac{1}{2} \ln \left(\frac{E_{3}+k_{3}}{E_{3}-k_{3}}\right) \\
& =\ln \left(\frac{E_{3}+k_{3}}{\bar{m}_{3}}\right)=\ln \left(\frac{\bar{m}_{3}}{E_{3}-k_{3}}\right) \\
& =\tanh ^{-1}\left(\frac{k_{3}}{E_{3}}\right)
\end{aligned}
$$

and sinular formulas hold for $y, y_{1}$ and $y_{2}$.

The four-momentum conservation is expressed by

$$
\begin{aligned}
0 & =\vec{q}_{1}+\vec{q}_{2}+\vec{q}_{3} \\
0 & =k_{1}+k_{2}+k_{3} \\
& =\tilde{m}_{1} \sinh y_{1}+\tilde{m}_{2} \sinh y_{2}+\tilde{m}_{3} \sinh y_{3} \\
\sqrt{s} & =2 E=E_{1}+E_{2}+E_{3} \\
& =2 m \cosh y \\
& =\tilde{m}_{1} \cosh y_{1}+\tilde{m}_{2} \cosh y_{2}+\tilde{m}_{3} \cosh y_{3}
\end{aligned}
$$

where $s$ is the square of the CM energy. Eq. (5c) implies that

$$
\tilde{m}_{1} e^{y_{1}}+\tilde{m}_{2} e^{y_{2}}+\tilde{m}_{3} e^{y_{3}}=\bar{m}_{1} e^{-y_{1}}+\bar{m}_{2} e^{-y_{2}}+\bar{m}_{3} e^{-y_{3}}
$$

which can be used to write energy conservation as

$$
\begin{aligned}
2 m \cosh y & =\tilde{m}_{1} e^{y_{1}}+\tilde{m}_{2} e^{y_{2}}+\tilde{m}_{3} e^{y_{3}} \\
& =\tilde{m}_{1} e^{-y_{1}}+\tilde{m}_{2} e^{-y_{2}}+\tilde{m}_{3} e^{-y_{3}}
\end{aligned}
$$

In the DPE region, one must have $y, y_{1}$ and $-y_{2}$ large and positive. For example, at RHIC with $p \times p$ at $100 \mathrm{GeV} / \mathrm{c}$, the rapidities are approximately $y \simeq y_{1} \simeq-y_{2} \simeq 5.3$, while $y_{3}$ and $\tilde{m}_{3}$ of the central system must remain at moderate values, e.g. $\bar{m}_{3} \simeq 1 \rightarrow 10 \mathrm{GeV}$ and $\left|y_{3}\right|<1.5$ (which 'defines' the central systcm).

Energy conservation may thus be expressed as

$$
\sqrt{s} \simeq m e^{y} \simeq \bar{m}_{1} e^{y_{1}} \simeq \bar{m}_{2} e^{-y_{2}}
$$


Let $e$ be equal to $1-x_{F}$ where $x_{F}$ is the Feynmann $x$, i.e.

$$
\begin{aligned}
& p_{1}=\left(1-\epsilon_{1}\right) p \\
& p_{2}=\left(1-\epsilon_{2}\right) p
\end{aligned}
$$

In the DPE region, the $\epsilon$ 's are very small; in general it is necessary to retain ouly the first order in $\epsilon$ 's for most relationships. One notable exception occurs for those involving four-momentum transfers. They are defined as follows

$$
\begin{aligned}
& t_{1}=\left(p_{a}-p_{1}\right)^{2} \\
& t_{2}=\left(p_{b}-p_{2}\right)^{2}
\end{aligned}
$$

which are given by

$$
\begin{aligned}
-t_{1} & =-2 m^{2}+2\left(E_{a} E_{1}-p_{a} p_{1} \cos \alpha_{1}\right) \\
& \simeq-2 m^{2}+2\left(E E_{1}-p p_{1}\right)+2 p\left(p_{1}-\left|k_{1}\right|\right) \\
-t_{2} & =-2 m^{2}+2\left(E_{b} E_{2}-p_{b} p_{2} \cos \alpha_{2}\right) \\
& \simeq-2 m^{2}+2\left(E E_{2}-p p_{2}\right)+2 p\left(p_{2}-\left|k_{2}\right|\right)
\end{aligned}
$$

where $\alpha_{1}$ is the scattering angle (assumed to be small). The energies above can be approximated by

$$
\begin{aligned}
E & \simeq p+\frac{m^{2}}{2 p} \\
E_{1} & \simeq p\left(1-\epsilon_{1}\right)+\frac{m^{2}}{2 p}\left(1+\epsilon_{1}+\epsilon_{1}^{2}\right)
\end{aligned}
$$

and similarly for $E_{2}$. Note that terms up to second order in $\epsilon_{1}$ have been retained in the expression above. Substituting Eq. (12) into Eq. (11), one obtains

$$
\begin{aligned}
& -t_{1} \simeq\left(\epsilon_{1} m\right)^{2}+\left(1+\epsilon_{1}\right) q_{1}^{2} \\
& -t_{2} \simeq\left(\epsilon_{2} m\right)^{2}+\left(1+\epsilon_{2}\right) q_{2}^{2}
\end{aligned}
$$

where one has used approximate relationships 


$$
\begin{array}{r}
k_{1} \simeq\left(1-\epsilon_{1}\right) p-\left(1+\epsilon_{1}\right)\left(\frac{q_{1}^{2}}{2 p}\right) \\
-k_{2} \simeq\left(1-\epsilon_{2}\right) p-\left(1+\epsilon_{2}\right)\left(\frac{q_{2}^{2}}{2 p}\right)
\end{array}
$$

Note that $k_{2}$ is negative in our definition corresponding to $y_{2}<0$. Note also that the minimum values for $-t_{1}$ and $-t_{2}$ involve second order terms in the $\epsilon$ 's and hence can be ignored for most applications. However, photon exchanges involve $t^{-1}$ propagators in the amplitude, and the minimum values for $-t$ prevent two-photon exchange amplitudes from 'blowing up.'

Substituting Eq. (14) and Eq. (12) into Eqs. (5b) and (5d), one obtains

$$
\begin{aligned}
& \left(\epsilon_{1}-\epsilon_{2}\right) p \simeq k_{3}-\left(\frac{q_{1}^{2}-q_{2}^{2}}{2 p}\right) \\
& \left(\epsilon_{1}+\epsilon_{2}\right) p \simeq E_{3}
\end{aligned}
$$

which can be added and subtracted to give

$$
\begin{aligned}
& 2 p \epsilon_{1} \simeq E_{3}-k_{3}+\left(\frac{q_{1}^{2}-q_{2}^{2}}{2 p}\right) \\
& 2 p \epsilon_{2} \simeq E_{3}+k_{3}-\left(\frac{q_{1}^{2}-q_{2}^{2}}{2 p}\right)
\end{aligned}
$$

By multiplying the two relationships above, one obtains

$$
\tilde{m}_{3}^{2} \simeq(2 p)^{2} \epsilon_{1} \epsilon_{2}-\left(\frac{k_{3}}{p}\right)\left(q_{1}^{2}-q_{2}^{2}\right)
$$

Or, dropping the second-order terms, one gets the familiar result

$$
\bar{m}_{3}^{2} \simeq s \epsilon_{1} \epsilon_{2}
$$

which shows that the transverse mass of the central system is related to the product of two $\epsilon$ 's. One may introduce a unitless quantity $\tau=\bar{m}^{2} / s$. Then one has

$$
\tau_{3} \simeq \epsilon_{1} \epsilon_{2}
$$

From Eq. (5a), the effective mass of the central system is given ly,

$$
m n_{3}^{2} \simeq s \epsilon_{1} \epsilon_{2}-\left|\overrightarrow{q_{1}}+\vec{q}_{2}\right|^{2}
$$


The second term above can be ignored as long as the $q$ 's are sufficiently small

$$
\begin{aligned}
& q_{1} \ll \sqrt{s} \epsilon_{1} \\
& q_{2} \ll \sqrt{s} \epsilon_{2}
\end{aligned}
$$

For double-Pomeron processes, these conditions are met when the slopes of the $-t$ distributions are sufficiently large; two-photon productions involve photon propagators, so that the $q$ terms can always be ignored. For $p \times p$ at $100 \mathrm{GeV} / \mathrm{c}$, for example, one has typically $\epsilon \sim 0.03$ for $\bar{m}_{3} \sim 5 \mathrm{GeV}$. Suppose further that the slope of the $-t$ distribution is $b \simeq 10 \mathrm{GeV}^{-2}$; then $-t \sim 0.1(\mathrm{GeV} / \mathrm{c})^{2}$ and $q \sim 0.33 \mathrm{GeV} / \mathrm{c}$. Note that the minimum of $-t$ as given in Eq. (13) is small indeed and can be ignored in this approximation. It is seen that in this case Eq. (20) is satisfied, so that in most applications the transverse mass in Eq. (17) can be replaced by the mass itself.

The relationships Eq. (15) can be used to recast the rapidity of the central system [see Eq. (4)\}

$$
y_{3} \simeq \frac{1}{2} \ln \left(\frac{\epsilon_{1}}{\epsilon_{2}}\right)
$$

In the DPE region, the $\epsilon$ 's cannot be arbitrarily large; their upper limits are in fact given by the maximum allowed effective mass $m_{3}=m_{x}(\simeq 10 \mathrm{GeV})$ of the central system, as follows

$$
\epsilon_{x}=\sqrt{\tau_{x}}=\frac{m_{x}}{\sqrt{s}}
$$

For a given $m_{3}$ the minimum $\epsilon$ is then given by, from Eq. (18),

$$
\epsilon_{0}=\frac{\tau_{3}}{\epsilon_{x}}=\sqrt{\tau_{x}^{-}}\left(\frac{m_{3}}{m_{x}}\right)^{2}
$$

Therefore the absolute minimum of $\epsilon_{0}$ occurs for the minimum allowed effective mass $m_{3}=m_{0}(\simeq 1 \mathrm{GeV})$ in the above expression, corresponding to the minimum of $\tau_{3}$, i.e. $\tau_{0}=m_{0}^{2} / s$. For $\sqrt{s} \simeq 200 \mathrm{GeV}$, the maximum and minimum values of $\epsilon$ are 0.05 and $5 \times 10^{-4}$, respectively. For a given $m_{3}$, the minimum and maximum values of $y_{3}$ are given by, from Eqs. (17), (21) and (22),

$$
\begin{aligned}
& y_{0}=-\ln \left(\frac{m_{x}}{m_{3}}\right)=-\frac{1}{2} \ln \left(\frac{\tau_{x}}{\tau_{3}}\right) \\
& y_{x}=+\ln \left(\frac{m_{x}}{m_{3}}\right)=+\frac{1}{2} \ln \left(\frac{\tau_{x}}{\tau_{3}}\right)
\end{aligned}
$$


Note that $y_{0}>-2.3$ and $y_{x}<2.3$ for all $m_{3}$.

In practice, the rapidity $y_{3}$ of the central system may not be arbitrarily as large or small as given above, as its decay products then escape detection in a finite detector. Suppose, for example, that

$$
\left|y_{3}\right|<1
$$

for a detector to have $90 \%$ or better acceptance for the decay products. Then, from Eq. (4), one finds

$$
\left|k_{3}\right|<\left(\frac{e^{2}-1}{2 e}\right) \tilde{m}_{3} \simeq 1.18 \tilde{m}_{3}
$$

which shows that the maximum allowed longitudinal momentum of the central system is proportional to its mass-a sensible result. The allowed region of $y_{3}$ is illustrated in Fig. 2. The constraint of Eq. (25) implies that the difference between $\epsilon$ 's cannot be arbitrarily large; indeed, from Eq. (21), one obtains

$$
\frac{\epsilon_{1}}{\epsilon_{2}}=e^{ \pm 2}
$$

A more systematic approach to delineating the allowed DPE region requires introduction of 'rapidity gaps.' Define them as follows

$$
\begin{aligned}
& \Delta_{1}=y_{1}-y_{3} \\
& \Delta_{2}=y_{3}-y_{2}
\end{aligned}
$$

To progress further, approximate forms of the rapidity are necessary. Substituting Eq. (15) into Eq. (4), one obtains

$$
\begin{aligned}
& y_{3} \simeq \ln \left(\frac{\sqrt{s}}{\tilde{m}_{3}}\right)-\ln \left(\frac{1}{\epsilon_{1}}\right) \\
& y_{3} \simeq-\ln \left(\frac{\sqrt{s}}{\tilde{r}_{3}}\right)+\ln \left(\frac{1}{\epsilon_{2}}\right)
\end{aligned}
$$

The $k_{1}$ and $k_{2}$ as given by Eq. (14) can be used in the definitions of $y_{1}$ and $y_{2}$, identical to those of $E_{1}$. (4), to find

$$
\begin{aligned}
& y_{1} \simeq \ln \left(\frac{\sqrt{s}}{\bar{n}_{1}}\right) \\
& y_{2} \simeq-\ln \left(\frac{\sqrt{s}}{\tilde{m}_{2}}\right)
\end{aligned}
$$


These relationships show that the rapidity gaps assume elegantly simple forms, as follows

$$
\begin{aligned}
& \Delta_{1} \simeq \ln \left(\frac{1}{\epsilon_{1}}\right) \\
& \Delta_{2} \simeq \ln \left(\frac{1}{\epsilon_{2}}\right)
\end{aligned}
$$

They are related, through Eq. (18),

$$
\Delta_{1}+\Delta_{2} \simeq \ln \left(\frac{1}{\tau_{3}}\right)
$$

Note also, from Eq. (21),

$$
\Delta_{2}-\Delta_{1} \simeq 2 y_{3}
$$

From these, one can draw the allowed DPE region as shown in Fig. 3. Also shown in the figure is the region for good detector acceptance, e.g. $\left|y_{3}\right|<1$.

\section{Phase Space}

Consider production of $n$ particles in Reaction (1). The invariant phase-space element may be defined by

$$
d \rho(n)=\prod_{i=1}^{n}\left(\frac{d^{3} p_{i}}{2 E_{i}}\right) \delta^{(4)}\left(\sum_{i=1}^{n} p_{i}-p_{a}-p_{b}\right)
$$

which can be broken up into two pieces with an intermediate system 3 of mass $m_{3}$, as follows

$$
d \rho(123 ; 3 \rightarrow f)=d \rho(123) d m_{3}^{2} d \rho(3 \rightarrow f)
$$

where the central system 3 couples to $f=n-2$ particles. It can be shown easily that integration over the four variables

$$
\frac{d^{3} p_{3}}{2 E_{3}} d m_{3}^{2}
$$

eliminates the $\delta$-function contained in $d \rho(3 \rightarrow f)$ and Eq. (35) reduces to Eq. (34). The second phase-space element $d \rho(3 \rightarrow f)$ in Eq. (35) is appropriate for the sub-processes initiated by two Pomerons and two photons; as such it is absorbed into the cross sections for these sub-processes, and therefore it is dropped in what follows. 
The phase-space element can now be written, in terms of the rapidity variables,

$$
\begin{aligned}
d \rho=\frac{1}{8} & d^{2} \vec{q}_{1} d^{2} \vec{q}_{2} d^{2} \vec{q}_{3} d y_{1} d y_{2} d y_{3} d m_{3}^{2} \\
& \times \delta^{(2)}\left(\vec{q}_{1}+\vec{q}_{2}+\vec{q}_{3}\right) \\
& \times \delta\left(\tilde{m}_{1} \sinh y_{1}+\tilde{m}_{2} \sinh y_{2}+\tilde{m}_{3} \sinh y_{3}\right) \\
& \times \delta\left(\tilde{m}_{1} \cosh y_{1}+\tilde{m}_{2} \cosh y_{2}+\tilde{m}_{3} \cosh y_{3}-2 m \cosh y\right)
\end{aligned}
$$

Integrating over $d^{2} \overrightarrow{q_{3}}$, one eliminates the first $\delta$-function. Integrating over the $d y_{2}$ takes care of the second $\delta$-function but brings in a factor in the denominator

$$
\tilde{m}_{2} \cosh y_{2}
$$

and the third $\delta$-function is eliminated by integrating over $d y_{1}$ but with $y_{3}$ fixed. For the purpose, one needs, from Eq. (5c),

$$
\bar{m}_{1} \cosh y_{1}+\tilde{m}_{2} \cosh y_{2}\left[\frac{d y_{2}}{d y_{1}}\right]_{y_{3}}=0
$$

One obtains, finally,

$$
d \rho=\frac{d^{2} \vec{q}_{1} d^{2} \vec{q}_{2} d y_{3} d m_{3}^{2}}{8 \tilde{m}_{1} \tilde{m}_{2} \sinh \left(y_{1}-y_{2}\right)}
$$

In the DPE region, the factor in the denominator is neariy constant [see Eq. (8)],

$$
\tilde{m}_{1} \tilde{m}_{2} \sinh \left(y_{1}-y_{2}\right) \simeq \frac{1}{2} \bar{r}_{1} e^{y_{1}} \tilde{r}_{2} e^{-y_{2}} \simeq \frac{s}{2}
$$

so that

$$
d \rho \simeq \frac{1}{4} d^{2} \vec{q}_{1} d^{2} \vec{q}_{2} d y_{3} d \tau_{3}
$$

In certain applications, it is more convenient to re-express the last two variables in terms of the $\epsilon$ 's [see Eq. (18) and Eq. (21)],

$$
d y_{3} d \tau_{3} \simeq d \epsilon_{1} d \epsilon_{2}
$$

Note that the Jacobian is equal to one. Integrating over the azimuths for $\vec{q}_{1}$ and $\overrightarrow{q_{2}}$ and noting the relationships Eq. (13),

$$
\begin{aligned}
d \rho & \simeq\left(\frac{\pi^{2}}{4}\right) d t_{1} d t_{2} d y_{3} d \tau_{3} \\
& \simeq\left(\frac{\pi^{2}}{4}\right) d t_{1} d t_{2} d \epsilon_{1} d \epsilon_{2}
\end{aligned}
$$


It is seen that double-exchange processes involve four variables, provided that the phase space for decay of the central system is iutegrated over, except its mass. They may either be two $-t$ 's and two $\epsilon$ 's or two $-t$ 's and the $y$ and $\tau$ of the central system. In both cases, the invariant phase-space element has a constant and the same weighting factor. Note that the two $-t$ 's range from $\left(\epsilon_{1} m\right)^{2}$ or $\left(\epsilon_{2} m\right)^{2}$ [see Eq. (13)] to $\infty$. Allowed DPE regions in the space of $y_{3}$ vs $\tau_{3}$ or of $\epsilon_{1}$ vs $\epsilon_{2}$ are succinctly given in Figs. 2 and 3.

\section{Elastic and Total Cross sections}

This section is due mainly to F. Paige. The elastic and total cross sections for $p \times p$ are given in the context of the Regge phenomenology, and a prescription is given for going over to heavy-ion collisions. The signature factors are neglected in this and the next sections.

The $t$ distribution of $p \times p$ elastic scattering is given by, in the high energy limit,

$$
\frac{d \sigma_{e l}}{d t} \sim \frac{1}{s^{2}}\left|F_{e l}(t)\right|^{2}
$$

where $F_{e l}(t)$ is the invariant amplitude

$$
F_{e l}(t)=i \gamma_{p}^{2}(t)\left(\frac{s}{s_{0}}\right)^{\alpha(t)}
$$

with

$$
\gamma_{p}(t)=\beta e^{b t / 4}
$$

and $\alpha(t)$ is the Pomeron trajectory $(\alpha(t) \simeq 1)$ and $b$ is the usual slope of the $t$ distribution $\left(b \simeq 15 \mathrm{GeV}^{-2}\right.$ for $\left.\sqrt{s}=200 \mathrm{GeV}\right)$. Note that $s_{0}$ is the usual constant of Regge theory $\left(\sqrt{s_{0}}=1 \mathrm{GeV}\right)$, and $\beta$ is the $p$-p-Pomeron coupling constant independent of $t$ and is a unitless quantity. Integrating over $t$, one obtains

$$
\sigma_{e l} \sim \frac{\beta^{4}}{b}
$$

The optical theorem relates the total cross section to the imaginary part of the elastic scattering amplitude, which takes on the form, in the high energy limit,

$$
\sigma_{t} \sim \frac{1}{s} \operatorname{Im}\left[F_{e l}(0)\right]
$$


so that

$$
\sigma_{t} \sim \beta^{2}
$$

Both the total and elastic scattering cross sections represent areas; it is therefore reasunable to assume that both increase as $A^{2 / 3}$, when $p \times p$ is replaced with $A \times A$ where $A$ is the nucleon numbez of any heavy-ion species. This implies that

$$
\begin{gathered}
\beta^{2} \propto A^{2 / 3} \\
b \propto A^{2 / 3}
\end{gathered}
$$

This result follows only if the factorization holds for $A \times A$ elastic scattering. As Pomerons are supposed to couple to individual nucleii, multiple Pomeron exchanges should be significant, which breaks the factorization. However, the slope parameter is thought to be proportional to the area, and the the $A$ dependence given above should be correct even if the facorization fails.

The cross sections $\sigma(A A)$ for $A \times A$ may now be written, in terms of $\sigma(p p)$ for $p \times p$,

$$
\begin{aligned}
\sigma_{\ell}(A A) & =f(A) A^{2 / 3} \sigma_{t}(p p) \\
\sigma_{e l}(A A) & =f(A) A^{2 / 3} \sigma_{e l}(p p) \\
b_{e l}(A A) & =f(A) A^{2 / 3} b_{e l}(p p)
\end{aligned}
$$

The coefficient $f(A)$ allows for an additional slowly varying $A$ dependence. It may be set arbitrarily

$$
f(A)=1+\ln (A)
$$

so that $f(A)=2.4$ for $\alpha(A=4)$ and $f(A)=6.3$ for $A u(A=197)$. The following formulas are useful; according to Goulianos ${ }^{7}$, one may write

$$
\begin{aligned}
\sigma_{t}(p p) & \simeq 38.5 \mathrm{mb}, \quad \sqrt{s}=8 \rightarrow 20 \mathrm{GeV} \\
& \simeq 26.3 \mathrm{mb}+(2.33 \mathrm{mb}) \ln \left(\frac{s}{2 m}\right), \quad \sqrt{s}>20 \mathrm{GeV} \\
\sigma_{e l}(p p) & \simeq 0.175 \sigma_{t}+(29 \mathrm{mb})\left(\frac{2 m}{s}\right) \\
b=b_{e l} & \simeq 7.9+0.7 \ln \left(\frac{s}{2 m}\right) \quad\left(\mathrm{GeV}^{-2}\right)
\end{aligned}
$$


where $\sqrt{s}$ and $m$ are both given in $\mathrm{GeV}$. The cross sections are for $\alpha \times \alpha$ [see Ref. 11] at $\sqrt{s}=126 \mathrm{GeV}: \sigma_{t}(\alpha, \alpha)=(250 \pm 50) \mathrm{mb}, \sigma_{e l}(\alpha, \alpha)=(45 \pm 15)$ mb and $b_{e l}(\alpha, \alpha)=$ $(100 \pm 10) \mathrm{GeV}^{-2}$. According to the prescription Eq. (48), they are estimated to be 287 $\mathrm{mb}, 50 \mathrm{mb}$ and $86 \mathrm{GeV}^{-2}$, respectively. It is seen that the slope parameter is somewhat under-estimated by Eq. (48).

At $\sqrt{s}=200 \mathrm{GeV}$, one has $\sigma_{t}(p p) \simeq 50 \mathrm{mb}, \sigma_{e l}(p p) \simeq 8.7 \mathrm{mb}$ and $b_{e l} \simeq 15 \mathrm{GeV}^{-2}$. Then, for $A u \times A u$ at $100 \mathrm{GeV} / \mathrm{u}$, the cross sections are, from Eq. (48),

$$
\sigma_{t}(A u, A u) \simeq 11 \mathrm{~b}, \quad \sigma_{e l}(A u, A u) \simeq 1.9 \mathrm{~b}, \quad b_{e l}(A u, A u) \simeq 3.2 \times 10^{3} \mathrm{GeV}^{-2}
$$

These cross sections are not known experimentally; the actual values when available may very well deviate by a factor of 2 or 3 . One sometimes assumes, in the high energy limit,

$$
\sigma_{t}(A u, A u)=4 \pi R^{2}
$$

where

$$
R \simeq(1.2 \mathrm{fm}) A^{1 / 3}
$$

This gives $\sigma_{t}(A u, A u)=6.1 \mathrm{~b}$, to be compared to $11 \mathrm{~b}$ in Eq. (51).

\section{Double-Pomeron Exchange Processes}

The aouble-Pomeron $(D P)$ excliange amiplitude can be written

$$
F_{D P}(a b \rightarrow 123) \sim \gamma_{p}\left(t_{1}\right)\left[z_{1}\right]^{\alpha\left(i_{1}\right)} \gamma_{p}\left(t_{2}\right)\left[z_{2}\right]^{\alpha\left(t_{2}\right)} F_{P P}(3 \rightarrow f)
$$

where the invariant amplitude $F_{P P}(3 \rightarrow f)$ refers to a process Pomeron+Pomeron $(P P) \rightarrow$ $f$ for the central system 3 . The residue functions $\gamma_{p}$ are as given in Eq. $(43)$, and $z_{i}(i=1,2)$ is the cosine of the t-channel scattering angle, which is given by [see Ref. 12]

$$
\begin{aligned}
z_{i} & =\frac{t_{i}^{2}+t_{i}\left(2 s-3 m^{2}-s_{i}\right)}{\left[\lambda\left(t_{i}, m, m\right) \lambda\left(t_{i}, \sqrt{s_{i}}, m\right)\right]^{1 / 2}} \\
\lambda\left(t_{i}, m, m\right) & =t_{i}\left(t_{i}-4 m^{2}\right) \\
\lambda\left(t_{i}, \sqrt{s_{i}}, m\right) & =\left[t_{i}-\left(\sqrt{s_{i}}+m\right)^{2}\right]\left[t_{i}-\left(\sqrt{s_{i}}-m\right)^{2}\right]
\end{aligned}
$$


The sub-energies $s_{i}$ are given by

$$
\begin{aligned}
s_{1} & =\left(p_{2}+p_{3}\right)^{2}=s+m^{2}-2 \sqrt{s} E_{1} \\
& \simeq m^{2}+s \epsilon_{1} \\
s_{2} & =\left(p_{3}+p_{1}\right)^{2}=s+m^{2}-2 \sqrt{s} E_{2} \\
& \simeq m^{2}+s \epsilon_{2}
\end{aligned}
$$

It is seen that, neglecting a $t_{i}$ dependence (which is 'absorbed' into $\gamma_{p}$ ),

$$
z_{i} \rightarrow \frac{s}{s_{i}-m^{2}} \simeq \frac{1}{\epsilon_{i}}
$$

With this, the amplitude assumes a simple form, using Eq. (43),

$$
F_{D P}(a b \rightarrow 123) \sim \beta^{2} \exp \left[b\left(t_{1}+t_{2}\right) / 4\right]\left(\frac{1}{\epsilon_{1} \epsilon_{2}}\right) F_{P P}(3 \rightarrow f)
$$

The total cross section for the sub-process Pomeron - Pomeron $(P P) \rightarrow f$ is expressed as follows [see Eq. (35)]

$$
\sigma_{P P}\left(m_{3}^{2}\right) \sim \frac{1}{m_{3}^{2}} \int\left|F_{P P}(3 \rightarrow f)\right|^{2} d \rho(3 \rightarrow f)
$$

where the flux factor is evaluated in the limit of zero mass. The differential cross section for the double-Pomeron exchange process now takes on the form

$$
\frac{d^{4} \sigma_{D P}}{d t_{1} d t_{2} d \epsilon_{1} d \epsilon_{2}} \sim \beta^{4} \exp \left[b\left(t_{1}+t_{2}\right) / 2\right]\left(\frac{m_{3}}{\epsilon_{1} \epsilon_{2}}\right)^{2} \sigma_{P P}\left(m_{3}^{2}\right)
$$

It is seen that the slope of the $t_{i}$ distribution is one half that of the elastic scattering. Integrating over $t_{1}$ and $t_{2}$ and noting Eq. (17) and Eq. (39), one obtains

$$
\frac{d^{2} \sigma_{D P}}{d m_{3}^{2} d y_{3}} \sim\left(\frac{\beta^{4}}{b^{2}}\right)\left(\frac{1}{m_{3}^{2}}\right) \sigma_{P P}\left(m_{3}^{2}\right)
$$

The total $P P$ cross section is thought to be nearly constant and is given by, according to Streng ${ }^{13}$,

$$
\sigma_{P F} \simeq 140 \mu \mathrm{b}
$$

This shows that the $D P$ cross section has roughly a $r n_{3}^{-2}$ dependence.

It is important to compare the $D P$ cross section $\mathrm{Eq}$. (61) to the elastic cross section Eq. (44). Because of independence of $t_{1}$ and $t_{2}$, the $D P$ cross section has a $b^{-2}$ (lependence. 
instead of a simple $b^{-1}$ behavior for the elastic cross section. From Eq. (47), one may now estimate the $D P$ cross section in heavy-ion collisions

$$
\sigma_{D P}(A A)=f(A) \sigma_{D P}(p p)
$$

Therefore, the $D P$ cross section should remain constant to first order in $A$. The $D P$ cross section for heavy ions is not known experimentally. For $\alpha \times \alpha$ it is measured ${ }^{14}$ that $\sigma_{D P} \sim$ $(131 \pm 25) \mu \mathrm{b}$ for two 'central' tracks $(|\eta|<1)$, to be compared with $\sigma_{D P} \sim(11.7 \pm 3.0) \mu \mathrm{b}$ for $p \times p$ collisions $^{14}$. It appears that $f(A)$ as given in Eq. (49) may not provide enough 'boost.' However, one must be cautious in extrapolating cross sections from $\alpha$ to heavy ions such as $A u$. It may be safe to assume, in any event, that there exists a factor of 3 or 4 uncertainty in Eq. (63).

As pointed out in the previous section, the factorization may fail for Pomeron exchanges in heavy-ion collisions. If so, the relationship Eq. (63) is clearly not correct. According to J. Bjorken and A. Mueller (private communications), the $D P$ cross sections should increase slowly, dependent only on the circumference of the involved nucleii, so that

$$
\sigma_{D P}(A A) \propto A^{1 / 3}
$$

According to this, the $D P$ cross section for $A u \times A u$ should increase approximatcly by a factor of 6 over that for $p \times p$.

For an absolute estimate of the $D P$ cross section, one must turn to an entirely different technique. Here one follows the steps carried out by Belforte and Goulianos ${ }^{8}$. Under the assumption of factorization in $D P$ processes, $\mathrm{Chew}^{15}$ pointed out

$$
\sigma_{D P}=\frac{1}{\sigma_{t}} \sigma_{D 1} \sigma_{D 2}
$$

where $\sigma_{D i}$ is the $p p$ single-diffraction-dissociation cross section and is given by ${ }^{8}$

$$
\frac{\sigma_{D}}{d t d \epsilon}=a b_{D} \exp \left[b_{D} t\right]\left(\frac{1}{\epsilon}\right)
$$

where $a=0.68 \mathrm{mb}$ and $b_{D}$ is the slope of the $t$ distribution. The formula Eq. (60) singgests that $b_{D}=b_{e l} / 2$; however, a more realistic experimental value ${ }^{8}$ is $b_{D}=b_{e l} / 1.5$, so that 
$b_{D}=10 \mathrm{GeV}^{-2}$ if $b_{e l}=15 \mathrm{GeV}^{-2}$ for $\sqrt{s}=200 \mathrm{GeV}$. From Eq. (65) and Eq. (66), one obtains

$$
\frac{d^{4} \sigma_{D P}}{d t_{1} d t_{2} d \epsilon_{1} d \epsilon_{2}}=\frac{\left(a b_{D}\right)^{2}}{\sigma_{t}} \exp \left[b_{D}\left(t_{1}+t_{2}\right)\right]\left(\frac{1}{\epsilon_{1} \epsilon_{2}}\right)
$$

which can be integrated over $t_{1}$ and $t_{2}$ and recast with the aid of Eq. (39)

$$
\frac{d^{2} \sigma_{D P}}{d m_{3}^{2} d y_{3}}=\left(\frac{a^{2}}{\sigma_{t}}\right)\left(\frac{1}{m_{3}^{2}}\right)
$$

Note the similarity of this formula with Eq. (61).

Assume now that $m_{3}$ is allowed to range from $m_{0}=1 \mathrm{GeV}$ to $m_{x}=10 \mathrm{GeV}$ and that in addition one imposes the condition $\left|y_{3}\right|<1$ (see Fig.2). Then, one obtains integrating over $y_{3}$

$$
\begin{array}{rlrl}
\frac{d \sigma_{D P}}{d m_{3}^{2}} & =\left(\frac{a^{2}}{\sigma_{t}}\right)\left(\frac{2}{m_{3}^{2}}\right), & & m_{0}<m_{3}<\frac{m_{x}}{e} \\
& =\left(\frac{a^{2}}{\sigma_{t}}\right)\left(\frac{2}{m_{3}^{2}}\right) \ln \left(\frac{m_{x}}{m_{3}}\right), & \frac{m_{x}}{e}<m_{3}<m_{x}
\end{array}
$$

The mass dependence of the $D P$ cross section is displayed in Fig. 4. The total $D P$ cross section is given by, upon integrating over $m_{3}$,

$$
\sigma_{D P} \simeq 85 \mu \mathrm{b}
$$

The DPE region of Fig. 2 results from certain conditions on $m_{3}$ and $y_{3}$; however, the DPE region is in practice not so clearly defined, depending in particular on the shape and nature of the detector and the decay product of the central system 3 . Suppose now that the condition $\left|y_{3}\right|<1$ prevails throughout the mass region $m_{0}=1 \mathrm{GeV}<m_{3}<m_{x}=10 \mathrm{GeV}$ under study. In this case, there exists a 'cut-off' mass $m_{c}=e m_{x} \gtrsim 27 \mathrm{GeV}$ [see Eq. (69a)]. Correspondingly, there follows a 'cut-off' on $\epsilon$; it is seen that, from $\mathrm{E}_{1}$. (22), one finds $\epsilon_{c} \gtrsim 0.14$. Under this condition the total $D P$ cross section becomes, from Eq. $(69 a)$,

$$
\sigma_{D P} \simeq 109 \mu \mathrm{b}
$$




\section{Two-Photon Processes}

The heavy-ion collisions represent a potential, prolific source of $\gamma \gamma$ processes as the

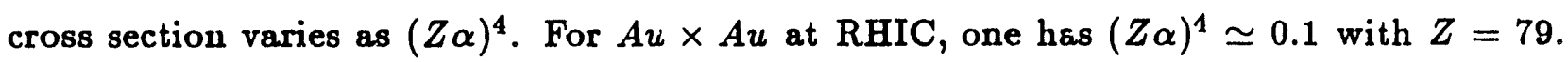
Therefore, the $\gamma \gamma$ processes should be, in principle, comparable to the $D P$ processes and in general the two reactions should interfere substantially.

Cahn and Jackson ${ }^{9}$ have recently calculated the $\gamma \gamma$ cross section in heavy-ion collisions within the framework of the Weizsäcker-Williams $(W W)$ approximation. The photon number distribution with energy $E_{\gamma}=\epsilon p$, carrying off a fraction of the initial beam momentum $p$, and an impact parameter $\vec{b}$ (defined in a plane perpendicular to the beam direction), is given by, ${ }^{16}$

$$
\frac{d^{3} N_{\gamma}}{d \epsilon d^{2} \vec{b}}=\left(\frac{Z^{2} \alpha}{\pi^{2}}\right)\left(\frac{1}{\epsilon b^{2}}\right) g(\epsilon m b) \equiv F_{\gamma}(\epsilon, \vec{b})
$$

where $m$ is the mass of the beam. Let $R$ be the radius of the nucleus [see Eq. (53)]. Then one has $b>R$. The function $g(x)$ is related to the modified Bessel function $K_{1}(x)$ through

$$
g(x)=\left[x K_{1}(x)\right]^{2}
$$

Integration over the impact parameter can be done in a closed form, using the formulas ${ }^{17}$

$$
\begin{aligned}
\int x K_{n}^{2}(x) d x & =\frac{x^{2}}{2}\left[K_{n}^{2}(x)-K_{n-1}(x) K_{n+1}(x)\right] \\
x K_{n+1}(x) & =x K_{n-1}(x)+2 n K_{n}(x)
\end{aligned}
$$

where $n$ is an integer. The following formulas are also useful

$$
\begin{aligned}
x \rightarrow 0, \quad K_{0}(x) & \rightarrow \ln \left(\frac{2}{x}\right)-\gamma \\
x \rightarrow 0, \quad K_{n}(x) & \rightarrow \frac{\Gamma(n)}{2}\left(\frac{2}{x}\right)^{n}, n>0 \\
x \rightarrow \infty, \quad K_{n}(x) & \rightarrow \sqrt{\frac{\pi}{(2 x)}} e^{-x}
\end{aligned}
$$

where $\gamma \simeq 0.5772$ is the Euler constant and $\Gamma(2)=\Gamma(1)=1$. From these it follows that

$$
\int_{a}^{\infty} \frac{d x}{x} g(x)=a K_{0}(a) K_{1}(a)-\frac{a^{2}}{2}\left[K_{1}^{2}(a)-K_{0}^{2}(a)\right] \equiv \tilde{g}(a)
$$


The photon number distribution as a function of $\epsilon$ can thus be written

$$
\epsilon \frac{d N_{\gamma}}{d \epsilon}=\left(\frac{2 Z^{2} \alpha}{\pi}\right) \tilde{g}(\epsilon m R)
$$

Let $F_{\gamma \gamma}\left(\epsilon_{1}, \epsilon_{2}\right)$ be the joint number distribution function of two photons carrying fractions $\epsilon_{1}$ and $\epsilon_{2}$ of the total momentum of each nucleus. It is given by integrating over the impact-parameter space a product of two Eq. (72)'s times a step function which ensures the the two beams do not 'overlap'

$$
F_{\gamma \gamma}\left(\epsilon_{1}, \epsilon_{2}\right)=\int d \vec{b}_{1} \int d \vec{b}_{2} F_{\gamma}\left(\epsilon_{1}, \vec{b}_{1}\right) F_{\gamma}\left(\epsilon_{2}, \vec{b}_{2}\right) \theta\left(\left|\vec{b}_{1}-\vec{b}_{2}\right|-2 R\right)
$$

The above step function can be recast as follows: one of the two azimuths for $\vec{b}$ 's can be integrated over immediately; the second one can be set to be the angle between $\vec{b}$ 's. Then the resulting $\Theta$-function, which ranges from 0 to 1 , assumes the form

$$
\begin{aligned}
\Theta\left(u_{1}, u_{2}\right) & =1, & & \left|u_{1}-u_{2}\right|>1 \\
& =1-\frac{1}{\pi} \arccos \left(\frac{u_{1}^{2}+u_{2}^{2}-1}{2 u_{1} u_{2}}\right), & & \left|u_{1}-u_{2}\right|<1
\end{aligned}
$$

where $u_{1}=b_{1} /(2 R)$ and $u_{2}=b_{2} /(2 R)$.

The effective differeatial luminosity to produce a central system of $m_{3}$ is, from Eq. (18),

$$
\frac{d \mathcal{L}}{d \tau_{3}}=\int d \epsilon_{1} d \epsilon_{2} F_{\gamma \gamma}\left(\epsilon_{1}, \epsilon_{2}\right) \delta\left(\tau_{3}-\epsilon_{1} \epsilon_{2}\right)
$$

In their paper they have performed integration over the entire kinematically allowed region of phàse space; however, one must in practice restrict the region of integration to that of the DPE region. For this purpose it is better to recast the variables into mass $m_{3}$ and rapidity $y_{3}$ of the central system. From Eq. (39), one may write, dropping the $\delta$-function,

$$
\frac{d \mathcal{L}}{d \tau_{3}}=\int d y_{3} F_{\gamma \gamma}\left(\epsilon_{1}, \epsilon_{2}\right)
$$

The $\epsilon$ 's are expressed, from Eq. (18) and Eq. (21),

$$
\epsilon_{1}=\sqrt{\tau_{3}} e^{y_{3}}, \quad \epsilon_{2}=\sqrt{\tau_{3}} e^{-y_{3}}
$$

An example of the allowed DPE region in the space of $\tau_{3}$ and $y_{3}$ is given in Fig. 2. 
They point out that the differential luminosity function can be expressed in terms of a single universal function $\xi(z)$. It involves a single variable $z$ given by

$$
z=2 m_{3} R m / \sqrt{s}=2 R m \sqrt{\tau_{3}}
$$

The function is defined via

$$
\tau_{3} \frac{d \mathcal{L}}{d \tau_{3}}=\mathcal{L}_{0} \xi(z)
$$

where

$$
\mathcal{L}_{0}=\frac{16 Z^{4} \alpha^{2}}{3 \pi^{2}}
$$

The universal function may be written

$$
\begin{aligned}
\xi(z) & =\frac{3}{4} \int d y_{3} G\left(\epsilon_{1}, \epsilon_{2}\right) \\
G\left(\epsilon_{1}, \epsilon_{2}\right) & =\int_{R}^{\infty} \frac{d b_{1}}{b_{1}} \int_{R}^{\infty} \frac{d b_{2}}{b_{2}} g\left(\epsilon_{1} m b_{1}\right) g\left(\epsilon_{2} m b_{2}\right) \Theta\left(\frac{b_{1}}{2 R}, \frac{b_{2}}{2 R}\right)
\end{aligned}
$$

It is seen, from Eqs. (82) and (83), that the arguements of the $g$-functions may be expressed in terms of the variables $u$ and $z$, so that the $G\left(\epsilon_{1}, \epsilon_{2}\right)$ function then depends on just two variables $z$ and $y_{3}$ through

$$
G\left(\epsilon_{1}, \epsilon_{2}\right)=\int_{\frac{1}{2}}^{\infty} \frac{d u_{1}}{u_{1}} \int_{\frac{1}{2}}^{\infty} \frac{d u_{2}}{u_{2}} g\left(u_{1} z e^{y_{3}}\right) g\left(u_{2} z e^{-y_{3}}\right) \Theta\left(u_{1}, u_{2}\right)
$$

Therefore, the function $\xi(z)$ as defined in Eq. (86a) depends indeed only on $z$ if the integration over $y_{3}$ in Eq. (86) is performed over the limits [see Eq. (24)]

$$
-\ln \left(\frac{z_{x}}{z}\right)<y_{3}<+\ln \left(\frac{z_{x}}{z}\right)
$$

where $z_{x}$ is the $z$ with $m_{3}=m_{x}$. Note that the limits on $y_{3}$ themselves depend on $z$. Of course, the function $\xi(z)$ depends also on $z_{x}$; however, its dependence turns out to be small for a sufficiently large value of $z_{x}$, i.e. $z_{x}>10$.

If the $\Theta$ function in Eq. (86b) is dropped from Eq. (87), then one can perform the required integrations separately. One obtains, from Eq. (76),

$$
\xi(z)=\frac{3}{4} \int d y_{3} \bar{g}\left(\frac{1}{2} z e^{y_{3}}\right) \bar{g}\left(\frac{1}{2} z e^{-y_{3}}\right)
$$


so that the function $\xi(z)$ is conveniently given through a single integration over $y_{3}$. Cahn and Jackson point out the effect of including the $\Theta$ function in Eq. (86b) is roughly equivalent to increasing the lower limit on b's in Eq. (86b) by $R \rightarrow 1.15 . R$.

For small $z$ it is seen from Eq. (87) that the integrand consists only of the the $\Theta$. function, as the $g$-function approaches one. Without the damping factor in the $g$-function, the upper limits tend to infinity, making $\xi(z)$ blow up at $z=0$; with the area of integration increasing in the plane of $u_{1}$ vs $u_{2}$ in the limit of small $z$, the $\Theta$-function can now be replaced by 'unity.' If $z$ is small, one has, from Eq1s. (75a), (75b) and (76),

$$
\tilde{g}(z) \rightarrow \ln \left(\frac{\lambda}{z}\right)
$$

where $2 \lambda=1.362$. Performing the required integration over $\left|y_{3}\right|<y_{x}$ on the factorized form Eq. (89), one finds

$$
\xi(z) \simeq \frac{1}{2} y_{x}\left[3 \ln ^{2}\left(\frac{2 \lambda}{z}\right)-y_{x}^{2}\right]
$$

Setting $y_{x}=1$, one obtains, trivially,

$$
\xi(z) \simeq \frac{1}{2}\left[3 \ln ^{2}\left(\frac{2 \lambda}{z}\right)-1\right]
$$

valid for $z \lesssim 0.05(\xi(z)=15.4$ at $z=0.05)$. If one sets the limits of the $y_{3}$ integration to those of Eq. (88), the formula Eq. (91) is a badly behaved function of $z_{x}$ for moderately small values of $z$; for example, $\xi(z)$ becomes negative if $z_{x}>15$ for $z=0.05$, while it reaches its maximum if $z_{x}=2 \lambda$ for $z=0.05$. Instead, one needs to expand Eq. (91) in powers of $\ln (c / z)$ for an arbitrary constant $c$ and retain only the highest power term, to obtain the result

$$
\xi(z) \simeq \ln ^{3}\left(\frac{c}{z}\right)
$$

which is very similar to that given by Cahn and Jackson and valid for small values of $z$. The value of $c=1.418$ in Eq. (93) matches $\xi(z)=37.4$ at $z=0.05$.

The $\xi(z)$ as a function of $z$ is given in Fig. 5 for $A u \times A u$ at $100 \mathrm{GeV}$ per nucleon $[z=$ $m_{3} /(2.83 \mathrm{GeV})$ and $\left.z_{x} \simeq 3.54\right]$, after integrating over $y_{3}$ in the DPE region as delineated in Fig. 2. The drop-off of $\xi(z)$ for $z>1.3$ reflects diminishing phase space for $y_{3}$ for high $m_{3}$. Also shown in the figure is the curve obtained by Cahn and Jackson integrating over the entire kinematically allowed region of phase space $\left(z_{x}=10\right.$ or larger $)$. 
The cross section for the two-photon process in heavy-ion collisions is, by definition, a product of the luminosity function Eq. (81) and the $\gamma \gamma$ cross section

$$
\tau_{3} \frac{d \sigma_{W W}}{d \tau_{3}}=\tau_{3} \frac{d \mathcal{L}}{d \tau_{3}} \sigma_{\gamma \gamma}\left(m_{3}\right)
$$

or, in terms of the mass $m_{3}$, from Eq. (84),

$$
\frac{d \sigma_{w W}}{d m_{3}^{2}}=\mathcal{L}_{0} \xi(z) \frac{\sigma_{\gamma \gamma}\left(m_{3}\right)}{m_{3}^{2}}
$$

The $\gamma \gamma$ cross section is measured in the $e^{+} e^{-}$storage rings; it is small (a factor of $\alpha^{2}$ is involved) and has a slow mass dependence as given below ${ }^{18}$

$$
\sigma_{\gamma \gamma}\left(m_{3}\right) \simeq\left[300+\frac{270}{m_{3}(\mathrm{GeV})}\right] \mathrm{nb}
$$

where the constant term has been chosen to approximate the data points for $m_{3}$ from 1 to $6 \mathrm{GeV}$.

The $W W$ cross section as a function of $m_{3}$ is given in Fig. 6 for $A u \times A u$ at $100 \mathrm{GcV} / \mathrm{u}$ (per nucleon). Also shown in the figure is the curve corresponding to the $D P$ cross section. Integrating Eq. (95) over $m_{3}$ from 1 to $10 \mathrm{GeV}$ in the region of phase space of Fig.2, one finds

$$
\sigma_{W W} \simeq 1.14 \mathrm{mb}
$$

to be compared to $85 \mu \mathrm{b}$ [see Eq. (70)] for the $D P$ process. Suppose now that the limits on rapidity $\left|y_{3}\right|<1$ are independent of mass for $m_{3}$ from 1 to $10 \mathrm{GeV}$, corresponding to the 'cut-off' values $m_{c} \gtrsim 27 \mathrm{GeV}$ and $\epsilon_{c} \gtrsim 0.14$. The $W W$ cross section changes little; it is still approximately $1.15 \mathrm{mb}$ (see the upper solid curve for $m_{3}>5 \mathrm{GeV}$ in Fig. 6), to be compared to $109 \mu \mathrm{b}$ for the $D P$ cross section. If the original $\xi(z)$ of Cahn and Jackson is used in Eq. (95), the $W W$ cross section is found to be $1.56 \mathrm{mb}$ when integrated over 1 to $10 \mathrm{GeV}$ for $m_{3}$; this then is the 'theoretical' maximum cross section for two-photon processes in this mass range. It is seen that $\gamma \gamma$ events should drop off much sharper in $m_{3}$ than the double-Pomeron events, indicating that they are useful mainly for study of low masses involving $u, d$ and $s$ quarks.

It is of some interest to know how RHIC compares to LEP for two-photon physics. To this end, a short description is given here for two-photon processes appropriate for $e^{\prime} e^{-}$ 
colliders. The corresponding luminosity function $\mathcal{L}_{e}$ was first worked out by Low ${ }^{19}$ some thirty years ago. The photon number distribution from a single beam is given by, in the farniliar $W W$ approximation,

$$
\frac{d N_{\gamma}}{d \epsilon}=\left(\frac{\alpha}{\pi}\right) \ln \left(\frac{\sqrt{s}}{2 m_{e}}\right) \frac{1+(1-\epsilon)^{2}}{\epsilon} \equiv F_{\gamma}(\epsilon)
$$

where $\epsilon$ is once again the fractional energy loss of the beam being carried off by the photon. The luminosity function is then given, from Eq. (81),

$$
\frac{d \mathcal{L}_{e}}{d \tau_{3}}=\int d y_{3} F_{\gamma}\left(\epsilon_{1}\right) F_{\gamma}\left(\epsilon_{2}\right)
$$

where the $\epsilon$ 's are given in Eq. (82).

One obtains the luminosity function derived by Low if the maximum of $m_{3}$ is set equal to its kinematical limit $m_{3}=\sqrt{s}$, so that the rapidity $y_{3}$ now has the limits, from Eq. (22) and Eq. (24),

$$
-\ln \left(\frac{1}{\sqrt{\tau_{3}}}\right)<y_{3}<+\ln \left(\frac{1}{\sqrt{\tau_{3}}}\right)
$$

The integration can be performed readily, and the result is

$$
\tau_{3} \frac{d \mathcal{L}_{e}}{d \tau_{3}}=\mathcal{L}_{1} h\left(\tau_{3}\right)
$$

where

$$
\mathcal{L}_{1}=2\left(\frac{\alpha}{\pi}\right)^{2}\left[\ln \left(\frac{\sqrt{s}}{2 m_{e}}\right)\right]^{2}
$$

and

$$
h\left(\tau_{3}\right)=\left(2+\tau_{3}\right)^{2} \ln \left(\frac{1}{\sqrt{\tau_{3}}}\right)-\left(3+\tau_{3}\right)\left(1-\tau_{3}\right)
$$

A more realistic luminosity results if one limits the range of the rapidity to $\left|y_{3}\right|<1$ or $y_{x}=1$. Let $h_{1}\left(\tau_{3}\right)$ be the new $h$-function. It is given by

$$
h_{1}\left(\tau_{3}\right)=\left(2+\tau_{3}\right)^{2}+\left(e^{2}-\frac{1}{e^{2}}\right) \tau_{3}-2\left(e-\frac{1}{e}\right)\left(2+\tau_{3}\right) \sqrt{\tau_{3}}
$$

This is valid as long as $m_{3}$ is less than $\frac{1}{e} \sqrt{s}$; for $m_{3}$ above that, the $h_{1}$ is to be replaced by the $h$ of Eq. (103), as the limit $y_{x}$ on $\left|y_{3}\right|$ becomes smaller than 1 and is given insticad by Eq. (100). 
The two-photon cross section for $e^{+} e^{-}$collisions may now be written

$$
\frac{d \sigma_{e e}}{d m_{3}^{2}}=\mathcal{L}_{1} h_{1}\left(\tau_{3}\right) \frac{\sigma_{\gamma \gamma}\left(m_{3}\right)}{m_{3}^{2}}
$$

where $\sigma_{\gamma \gamma}$ is given in Eq. (96). The cross section $\sigma_{e e}$ is shown in Fig. 7. Assume $\sqrt{s}=$ $100 \mathrm{GeV}$ at LEP; then the total cross section with $y_{x}=1$ is, integrated over $m_{3}$ from 1 to $10 \mathrm{GeV}$ with $y_{x}=1$,

$$
\sigma_{e e} \simeq 9.85 \mathrm{nb}
$$

to be compared to $\sigma_{w w} \simeq 1.15 \mathrm{mb}$ for RHIC. If the $h$-function Eq. (103) of Low is used to calculate the cross section, one finds $\sigma_{e e} \simeq 30.1 \mathrm{nb}$. The luminosity at LEP is approximately $10^{31} \mathrm{~cm}^{-2} \mathrm{sec}^{-1}$ at $\sqrt{s}=100 \mathrm{GeV}$, whereas it is $10^{26} \mathrm{~cm}^{-2} \mathrm{sec}^{-1}$ for $A u \times A u$ at $100 \mathrm{GeV} / \mathrm{u}$ at $\mathrm{RHIC}$. This shows that the two-photon event rate for the two machines is approximately the same for a period of run of equal time. Fig. 7 shows the RHIC cross section scaled down by the luminosity ratio, which demonstrates that the event rate is higher at RHIC for mass less than $2 \mathrm{GeV}$.

\section{Acknowledgements}

The authors are indebted to F. Paige for several helpful discussions.

\section{DISCLAIMER}

\footnotetext{
This report was prepared as an account of work sponsored by an agency of the United States Government. Neither the United States Government nor any agency thereof, nor any of their employees, makes any warranty, express or implied, or assumes any legal liability or responsibility for the accuracy, completeness, or usefulness of any information, apparatus, product, or process disclosed, or represents that its use would not infringe privately owned rights. Reference herein to any specific commercial product, process, or service by trade name, trademark, manufacturer, or otherwise does not necessarily constitute or imply its endorsement, recommendation, or favoring by the United States Government or any agency thereof. The views and opinions of authors expressed herein do not necessarily state or reflect those of the United States Government or any agericy thereof.
} 


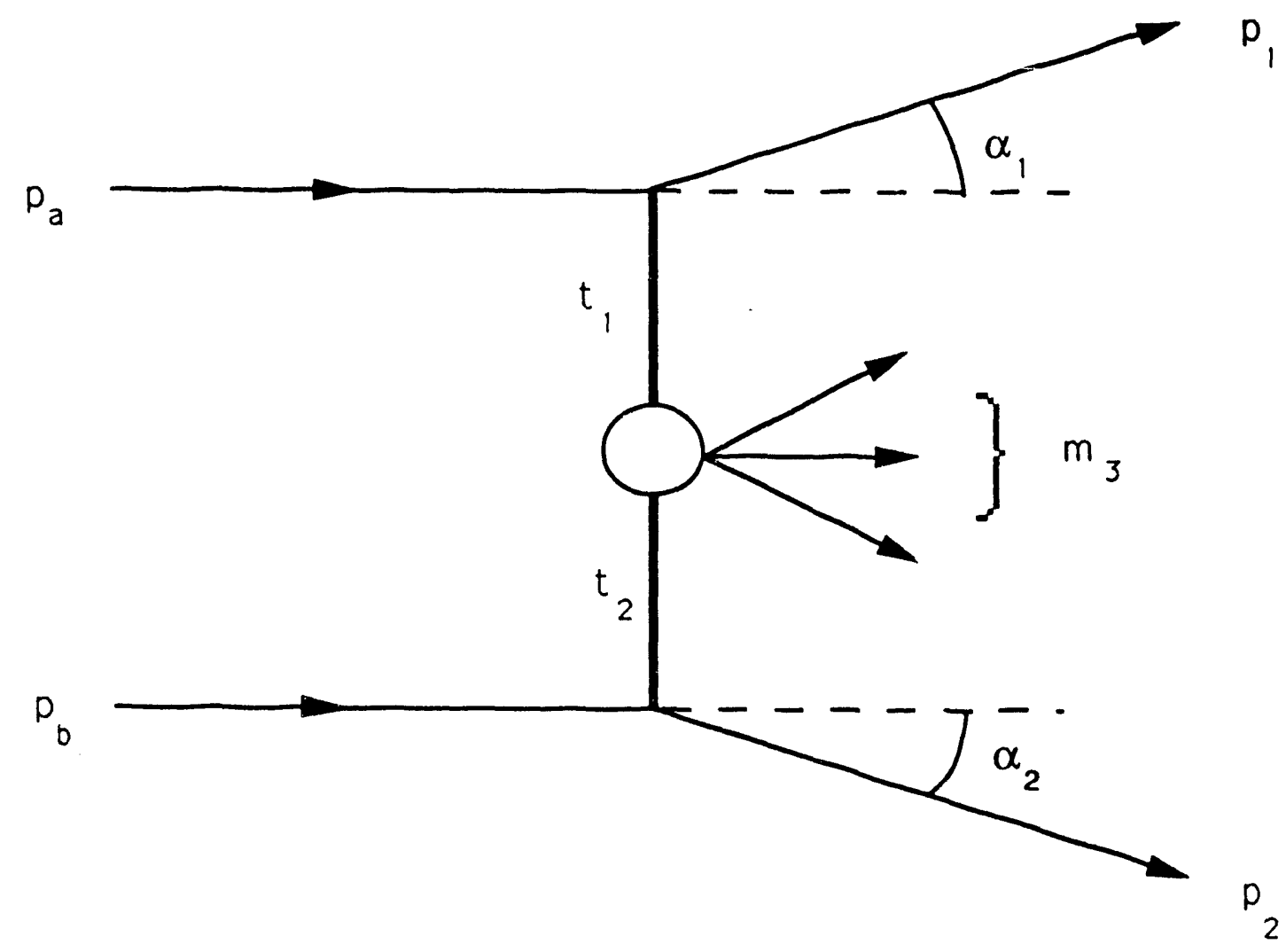

Figure 1. Double-Pomeron exchange diagram for production of a central system with mass $m_{3}$. The center-of-mass scattering angles are denoted $\alpha_{1}$ and $\alpha_{2}$. The fourmomentum transfers $t_{1}$ and $t_{2}$ are defined for particles $a$ to 1 and for particles $b$ to 2 . 


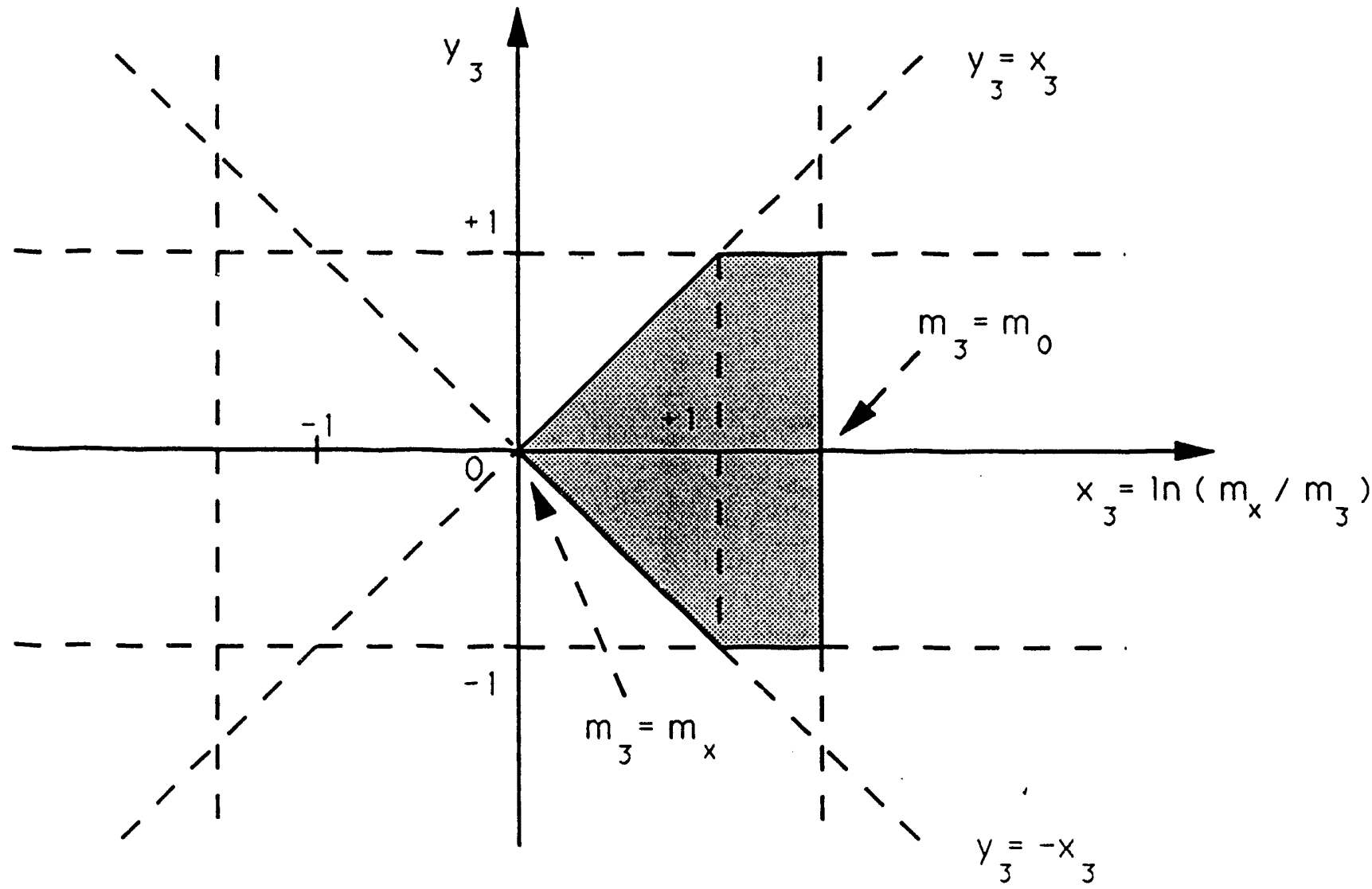

Figure 2. Allowed double-Pomeron exchange region for the central system with mass $m_{3}$, given in the space of its rapidity $y_{3}$ and $x_{3}=\ln \left(m_{x} / m_{3}\right)=(1 / 2) \ln \left(\tau_{x} / \tau_{3}\right)$. 


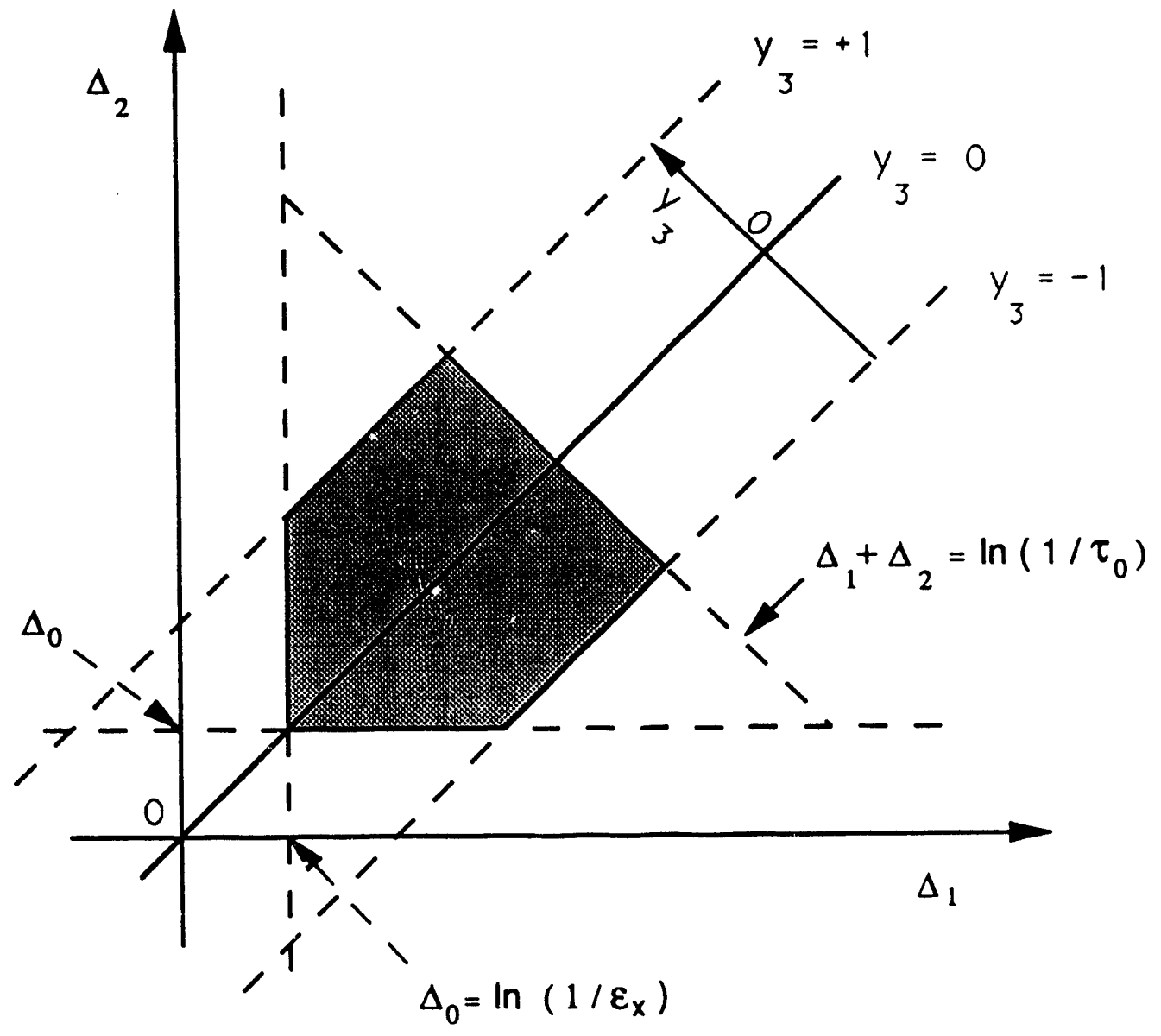

Figure 3. Allowed double-Pomeron exchange region for the central system with mass $m_{3}$, given in the space of rapidity gaps $\Delta_{1}$ and $\Delta_{2}$. 


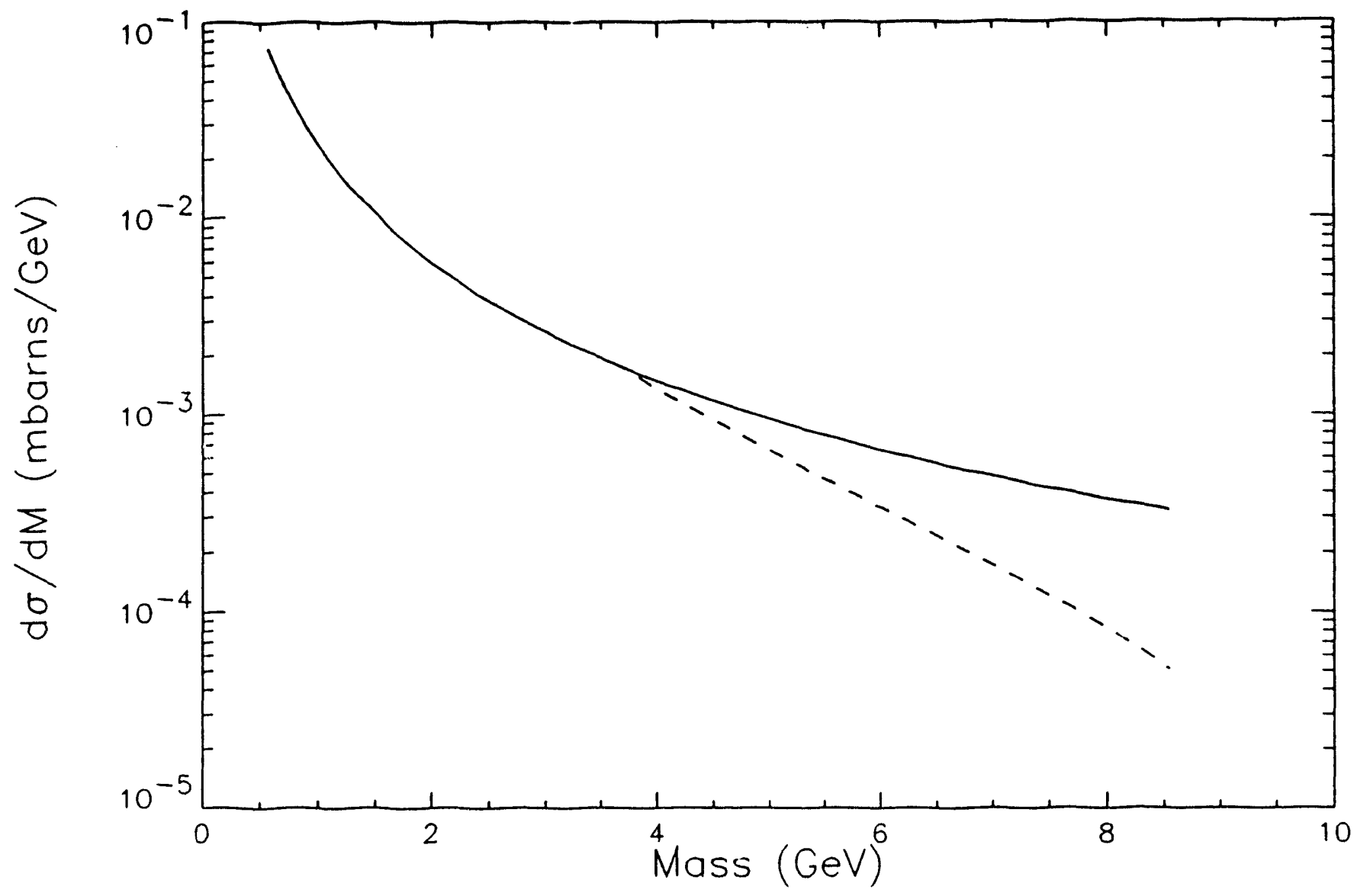

Figure 4. The cross section for the double-Pomeron exchange (DPE) process at RHIC ( $p \times p$ at $100 \mathrm{GeV} / \mathrm{c}$ ) as a funtion of mass $m_{3}$ of the central system for the DPE region as given in Fig. 2 (the dashed curve for $m_{3}>3.7 \mathrm{GeV}$ ). The upper solid curve results from setting $y_{x}=1$ for $m_{3}$ from 1 to $1 \% \mathrm{GeV}$, i.e. $m_{c}>27 \mathrm{GeV}$. 


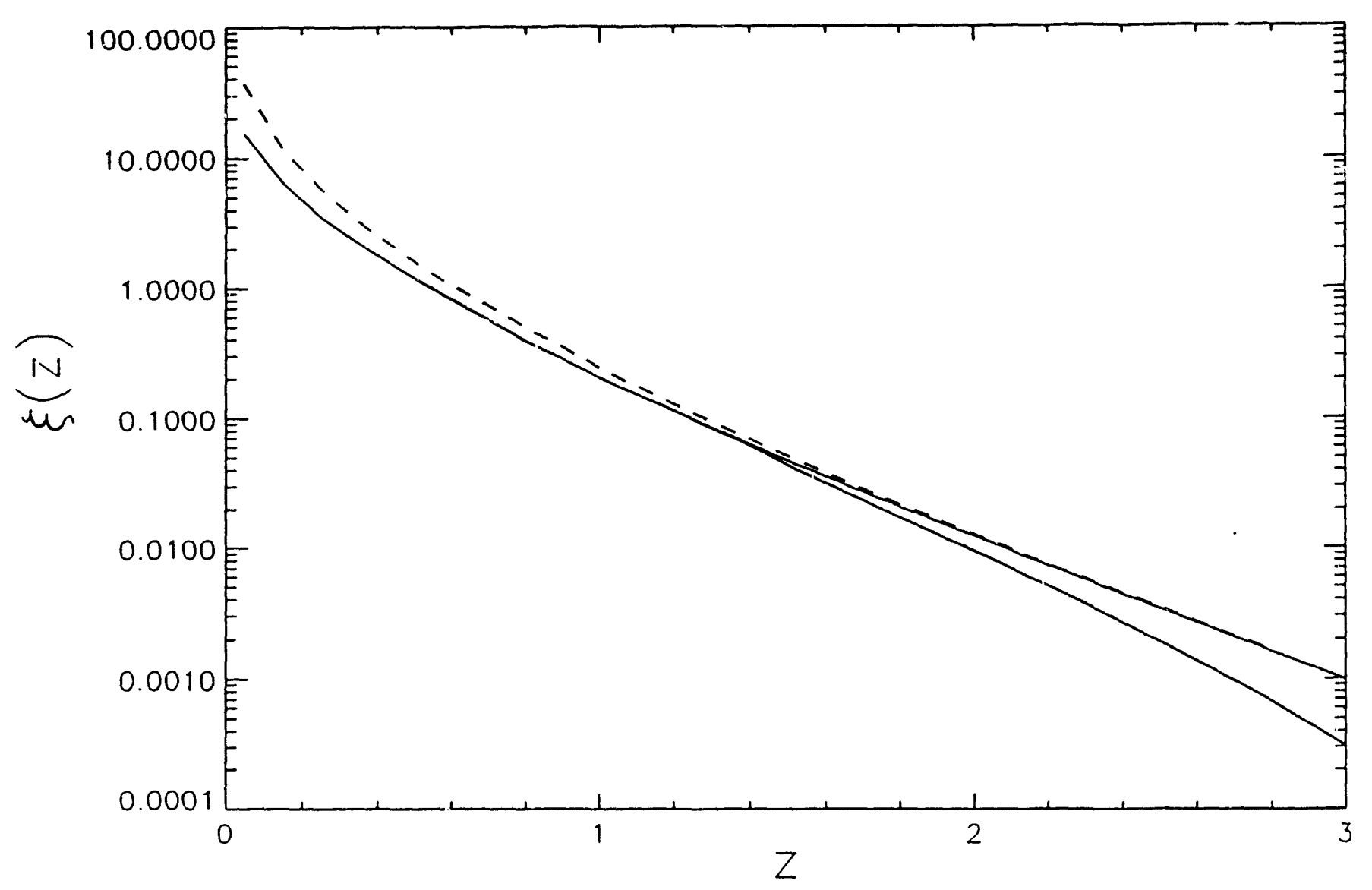

Figure 5. The universal function $\xi(z)$ for the region of phase space as given in Fig. 2. The upper solid curve for $z>1.3$ corresponds to setting $y_{x}=1$ up to $z=3$. The dashed curve is the original $\xi(z)$ as given in Cahn and Jackson ${ }^{9}$. 


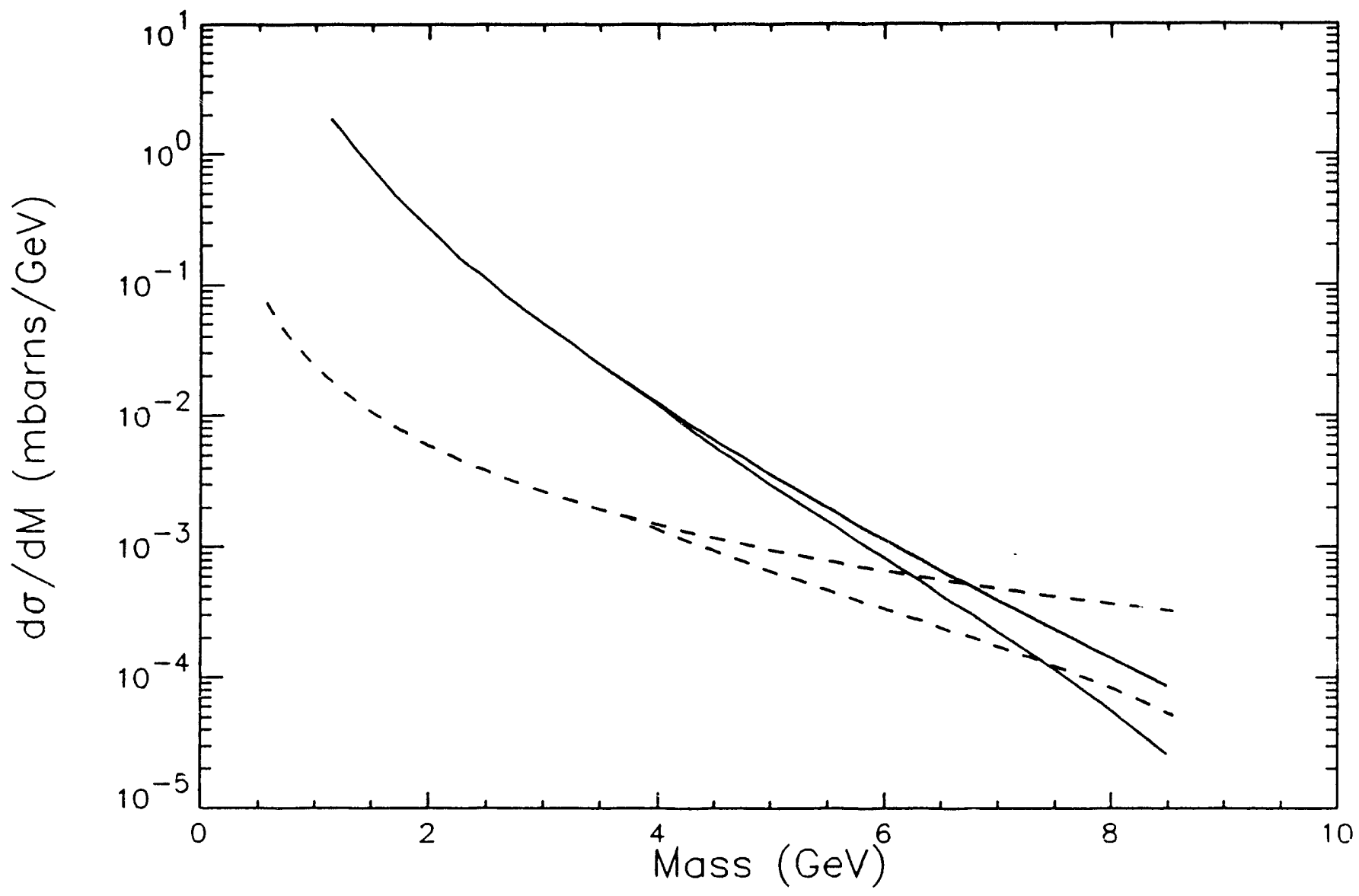

Figure 6. The cross section for the two-photon process at RHIC ( $A u \times A u$ at 100 $\mathrm{GeV} / \mathrm{u}$ ) as a function of mass $m_{3}$ of the central system for the region of phase space as given in Fig. 2. The upper solid curve for $m_{3}>3.7 \mathrm{GeV}$ corresponds to setting $y_{x}=1$ up to $m_{3}=10 \mathrm{GeV}$. For comparison the double-Pomeron cross section is also shown as a dotted curve. 


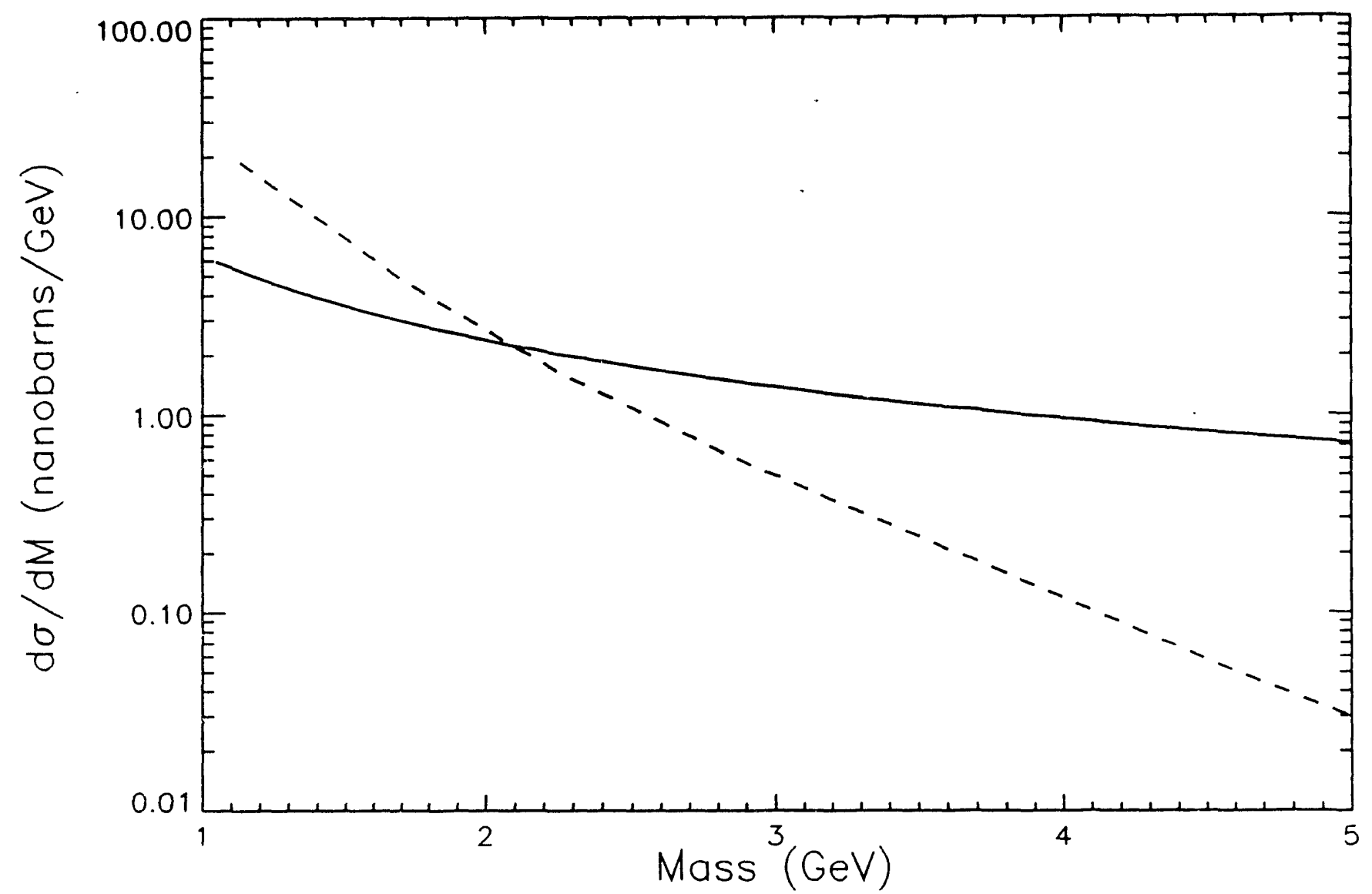

Figure 7. The two-photon cross section at LEP as a function of $m_{3}$ for $\sqrt{s}=$ $100 \mathrm{GeV}$ with $y_{x}=1$. Also shown is the cross section for RHIC scaled down by $10^{-5}$, the ratio of the luminosity at RHIC to that at LEP. 


\section{References}

1. T. Åkesson, et al., Nucl. Phys. B264, 154 (1986).

2. D. Drijard, et al., Nucl. Phys. B143, 61 (1978).

3. B. R. Desai, B. B. Shen and M. Jacob, Nucl. Phys. B142, 258 (1978).

4. H. De Kerret, et al., Phys. Lett. $\underline{68 \mathrm{~B}}, 385$ (1977).

5. M.-S. Chen and G. L. Kane, Phys. Lett. $\underline{60 B}, 192$ (1976).

6. J. Pumplin and F. S. Henyey, Nucl. Phys. B117, 377 (1976).

7. 'Diffractive Interactions of Hadrons at High Energies,' K. Goulianos, Physics Reports $\underline{101}, 169$ (1983).

8. 'Double-Pomeron Exchange at the Fermilab Collider,' S. Belforte and K. Goulianos, CDF Note No. 262.

9. R. N. Cahn and J. D. Jackson, LBL-28592-Rev (August 1990).

10. 'Kinematics, Phase Space and Phenomenological Amplitudes for Double-Pomeron Exchange Reactions,' S. U. Chung, BNL-OG 734 (1983).

11. M. Ambrosio, et al., Phys. Lett. 113B, 347 (1982).

12. 'An Introduction to Regge theory and high energy physics,' P.D.B. Collins, Cambridge University Press (1977), p. 21.

13. K.H. Streng, Pliys. Lett. 166B, 443 (1986).

14. V. Cavasinni, et al., Z. Phys. C28, 487 (1985).

15. D.M. Chew and G. F. Chew, Phys. Lett. $\underline{53 \mathrm{~B}}, 191$ (1974).

16. 'Classical Electrodynamics,' J. D. Jackson, John Wiley \& Sons (1975).

17. 'Table of Integrals, Series and Products-Corrected and Enlarged Edition,' I. S. Gradshteyn and I. M. Ryzhik, Academic Press (1980).

18. 'Two-Photon Physics,' H. Kolanoski and P. Zerwas, DESY $87-175$ (1987).

19. F. Low,Phys. Rev. 120,582 (1960). 

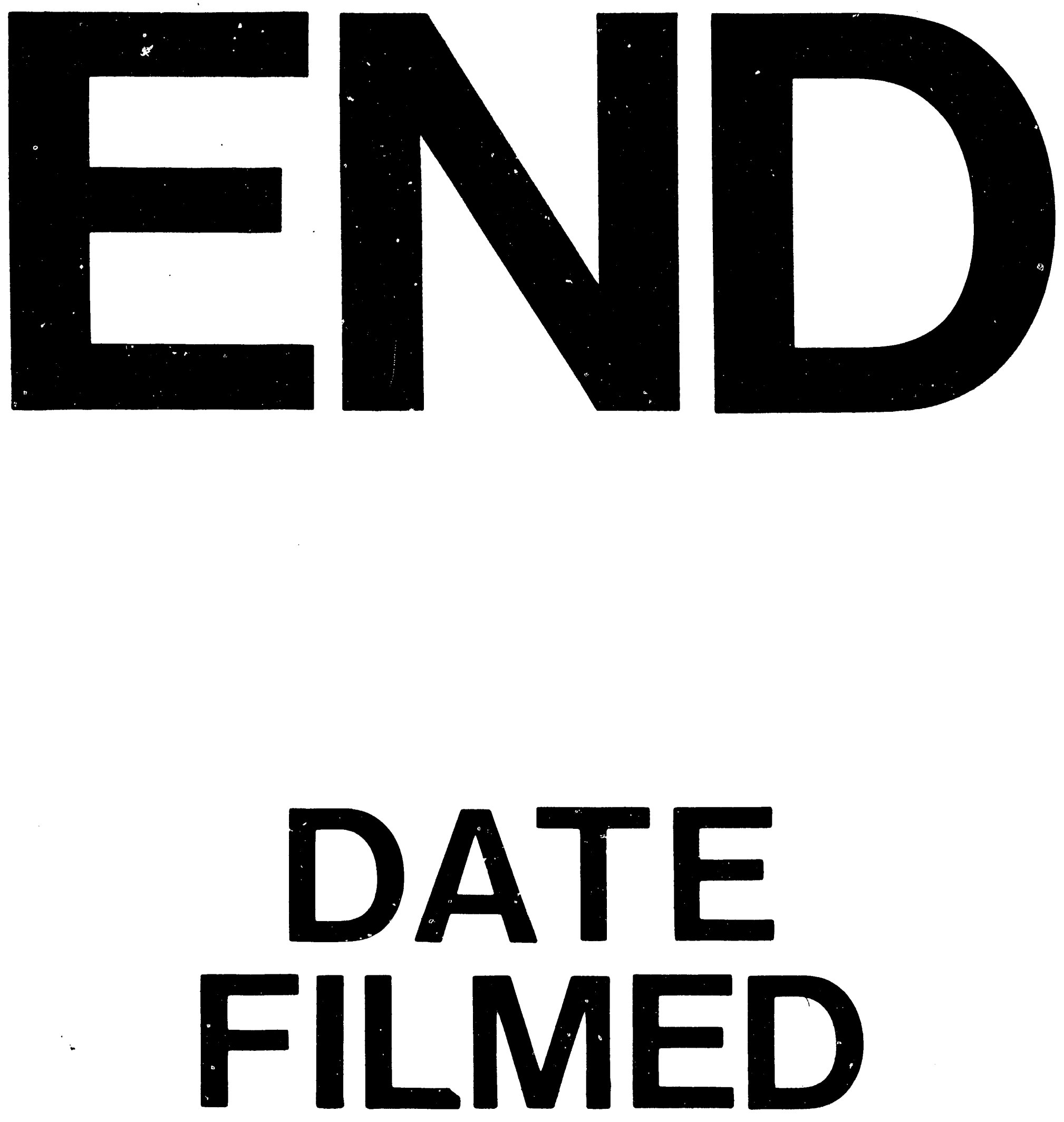

1

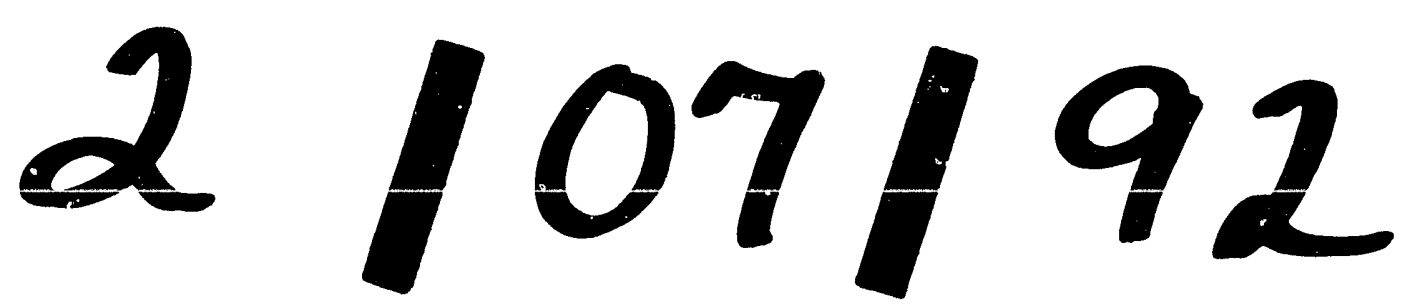


